\title{
Importance of the selection of feed tray location on the optimum design of a heterogeneous azeotropic distillation column with p-xylene feed impurity
}

\author{
I-Lung Chien ${ }^{\mathrm{a}^{*}}$, Hao-Yeh Lee ${ }^{\mathrm{b}}$, Tang-Kai Gau ${ }^{\mathrm{b}}$, and Hsiao-Ping Huang ${ }^{\mathrm{b}}$ \\ ${ }^{a}$ Department of Chemical Engineering, National Taiwan University of Science and \\ Technology, Taipei 106, Taiwan. \\ ${ }^{b}$ Department of Chemical Engineering, National Taiwan University, Taipei 106, Taiwan.
}

\begin{abstract}
In the production of aromatic acid, such as terephthalic acid, tiny amounts of one reactant (in this study, p-xylene) may also enter into the acetic acid dehydration column through the feed stream. In this work, the process design flowsheets with and without this tiny impurity are both considered. For the case with this tiny impurity in the feed stream, a side stream is necessary to purge out this impurity, otherwise, accumulation of this impurity will occur inside the column. If only optimum side-stream location and its flow rate are considered in the optimization search, it is found that the TAC (total annual cost) and the operating cost of this acetic acid dehydration column become much higher by just adding extremely small amount of p-xylene in the feed stream. With careful selection of the feed tray location, significant TAC as well as energy savings for the operation of this column can be realized.
\end{abstract}

Keywords: Acetic Acid, Heterogeneous Azeotropic Distillation, Column Design, Side Stream, Feed Tray Location

\section{Introduction}

Acetic acid (HAc) dehydration is an important operation in the production of aromatic acid, such as terephthalic acid, or in the manufacture of cellulose acetate. To make the separation easier, an entrainer is often introduced into the system. In a review paper, Othmer [1] described an azeotropic distillation system containing a dehydrating column, a decanter, and a water column for the separation of HAc and water. The entrainer used before 1932 was ethylene dichloride, and later n-propyl acetate and nbutyl acetate were used to reduce the organic reflux and heat duty used in the dehydrating column. In a paper by Pham and Doherty [2], examples of using ethyl acetate (cf. Tanake and Yamada [3]), n-propyl acetate (cf. Othmer [4]), or n-butyl acetate (cf. above [3] and [4]) as the entrainer were listed in a table of examples of heterogeneous azeotropic separations. Siirola [5] uses HAc dehydration as an example to demonstrate a systematic process synthesis technique to the conceptual design of a process flowsheet. Ethyl acetate was used as the entrainer in that paper to design a complete HAc dehydration process with multiple-effect azeotropic distillation and heat integration. Wasylkiewicz et al. [6] proposed using a geometric method for the optimum design of a HAc dehydrating column with n-butyl acetate as the entrainer. Recently, Chien, et al. [7] studied the design and control of HAc dehydration system via heterogeneous azeotropic distillation. A suitable entrainer of isobutyl acetate (IBA) was selected from three candidate acetates by TAC analysis.

\footnotetext{
* Corresponding author. I-Lung Chien, Tel: 886-2-2737-6652; Fax: 886-2-2737-6644., E-mail: chien@,ch.ntust.edu.tw
} 
In the previous studies, only three components (HAc, $\mathrm{H}_{2} \mathrm{O}$, and the entrainer) in the heterogeneous distillation column were focused. However, in the production of aromatic acid, such as terephthalic acid, tiny amounts of one reactant may also enter into the acetic acid dehydration column through the feed stream. Chien, et al. [8] studied the design and operation of an industrial column for HAc dehydration with five feed streams. The entrainer used for this industrial column to aid the separation is also IBA. In that paper, optimum design of the side-stream location and its flow rate were performed and an automatic purging strategy was proposed to prevent accumulation of an impurity (not specified for proprietary reason) inside the column. However, the effect of changing the feed tray location was not investigated.

In this work, HAc dehydration column via heterogeneous azeotropic column system with and without the impurity in the feed stream will be thoroughly studied. The entrainer used in the study is also isobutyl acetate and the impurity in the feed stream is assumed to be p-xylene which is a reactant commonly used in the terephthalic acid plant. Three optimum design processes will be studied. The first one assumes that the tiny amount of p-xylene is not considered in the feed stream. The optimized design and operating variables include: the column total stages, feed tray location, and the aqueous reflux ratio. The column total stages and the feed tray location obtained from this optimum search will be used in the second study with tiny amounts of p-xylene entering into the column through the feed stream. The optimal side-stream location and its flow rate will be investigated. In the third design also with the impurity in the feed stream, besides the side-stream location and its flow rate, the feed tray location will also be adjusted in this case. The results of the above three cases will be compared.

\section{Steady-state design with no feed impurity}

In this study, the feed stream is assumed to contain equal molar of acetic acid (HAc) and water $\left(\mathrm{H}_{2} \mathrm{O}\right)$. The entrainer (isobutyl acetate, IBA) is introduced into the column through organic reflux stream. Because the addition of the entrainer IBA will form a heterogeneous azeotrope with minimum temperature of the system, thus this azeotrope will ideally go out of the system from column top. After cooling of this stream to $40^{\circ} \mathrm{C}$ at decanter, this stream will naturally split into two liquid phases. The organic phase containing mostly IBA can be refluxed back to the column. Part of the aqueous phase is also refluxed back to the column for high purity reason with the rest going out of the system as waste water stream. The process flowsheet can be seen in Figure 1. The product specifications are $99.9 \mathrm{~mol} \%$ of HAc to be recovered from the column bottoms and high purity water stream containing only $0.1 \mathrm{~mol} \% \mathrm{HAc}$ to be withdrawn from the decanter aqueous outlet stream. In the process simulation, the bottom product specification is set by varying the reboiler duty and the aqueous outlet product specification is set by varying the entrainer makeup flow rate.

There are three design and operating variables in this flowsheet which can be optimized. They are: the column total stages, feed tray location, and the aqueous reflux fraction. The optimization procedure is to find the minimized total annual cost (TAC) at particular column total stages by varying the feed tray location and aqueous reflux fraction. For each simulation run, process simulation tool of Aspen Plus [9] was used to obtain the simulation result. Figure 2 shows the optimized result with the column total stages fixed at 39 (not counting reboiler). With the similar optimization search at other total stages, the overall minimized TAC can be found at column total stages at 39 with feed tray location at $9^{\text {th }}$ stage and aqueous reflux fraction set at 0.11 . The optimized base case condition is summarized in Figure 3. 


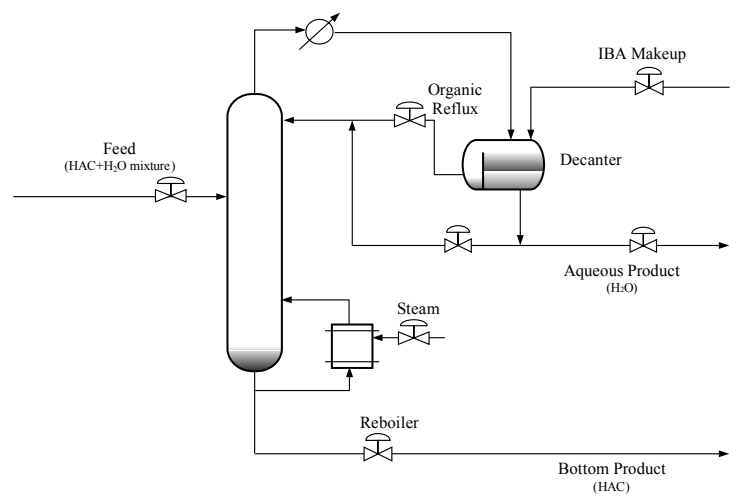

Fig. 1. Process flowsheet without feed impurity

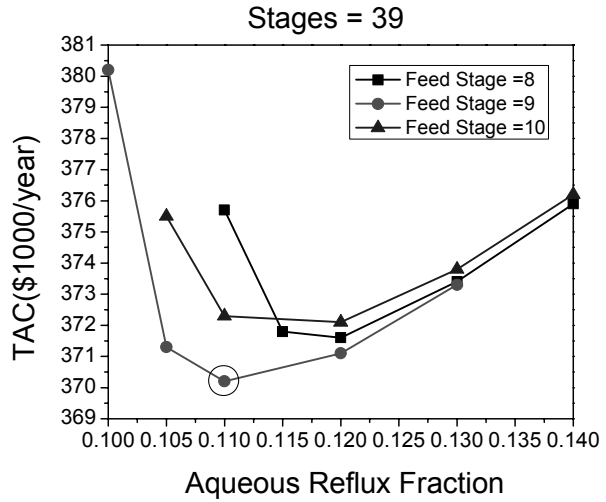

Fig. 2. Optimized results with column total stages at 39.

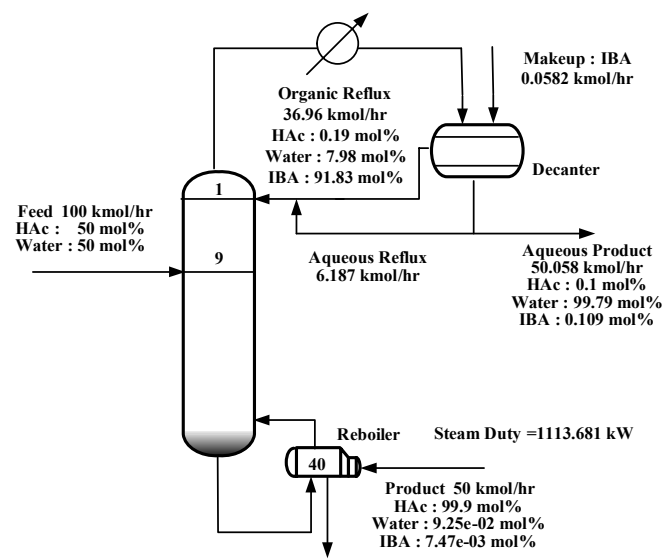

Fig. 3. Optimized base case condition for no impurity case. 


\section{Steady-state design with feed impurity}

\subsection{Side-Stream Location and Its Flow Rate as the Optimized Variables.}

In the production of aromatic acid, such as terephthalic acid, tiny amounts of one reactant (in this study, p-xylene) may also enter into this acetic acid dehydration column through the feed stream. Thus, a side stream is necessary to purge out this impurity, otherwise, accumulation of this impurity will occur inside the column. In this second case with feed impurity, feed composition of $50 \mathrm{~mol} \%$ water, $49.9 \mathrm{~mol} \%$ acetic acid, and $0.1 \mathrm{~mol} \% \mathrm{p}$-xylene is assumed. Intuitive design thinking is to just use the optimum total stages and feed tray location obtained from the above no impurity case and then investigating the optimal side-draw flow rate and side stream location via TAC analysis. The merit of this design thinking is that the same column design can be suited for both the no impurity case and with tiny impurity case.

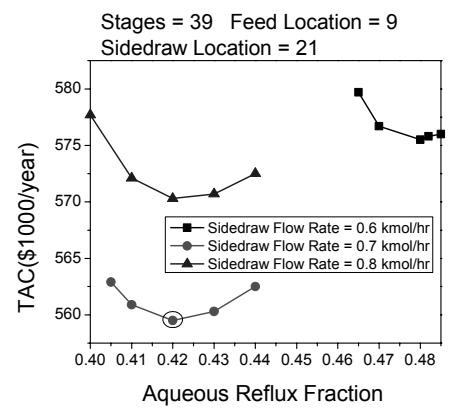

Fig. 4. Optimized results with sidedraw location at $21^{\text {st }}$ stage.

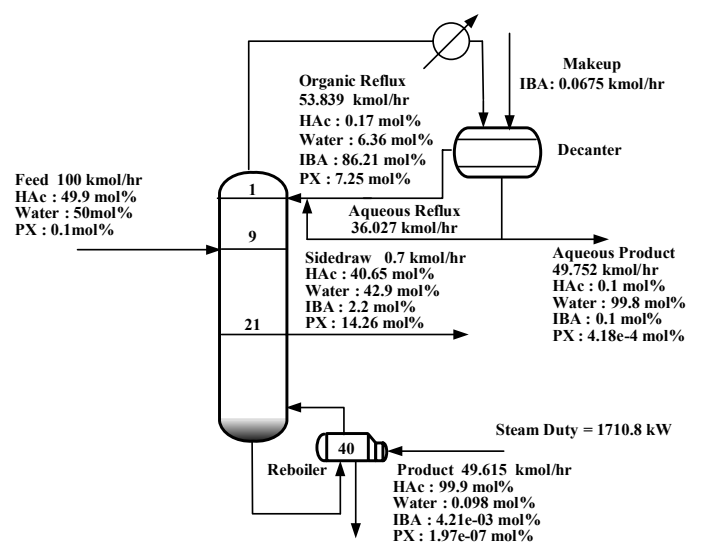

Fig. 5. Optimized base case condition with feed impurity (not changing feed tray location).

The optimization procedure is to fix the total stages at 39 (not counting reboiler), feed tray location at $9^{\text {th }}$ stage, and to vary the sidedraw location and sidedraw flow rate. Again, the bottom product specification is set by varying the reboiler duty and the aqueous outlet product specification is set by varying the entrainer makeup flow rate. For each simulation run, the aqueous reflux fraction gives the lowest TAC is selected. Figure 4 is an example of such simulation run with sidedraw location at $21^{\text {st }}$ stage. 
From the figure, aqueous reflux fraction of 0.42 and sidedraw flow rate of $0.7 \mathrm{kmol} / \mathrm{h}$ will be selected. Doing similar runs at other sidedraw location, the overall optimized result is found. The optimized base case condition is summarized in Figure 5.

Comparing the base case conditions of Figs. 3 and 5, it is found that the reboiler duty is changed from $1114 \mathrm{KW}$ to $1711 \mathrm{KW}$, a $54 \%$ increase in this energy cost. The TAC is changed from $\$ 370.2 \times 10^{3}$ to $\$ 559.5 \times 10^{3}$ (a $51 \%$ increase), and the operating cost is changed from $\$ 106.7 \times 10^{3}$ to $\$ 163.8 \times 10^{3}$ (a $54 \%$ increase). This shows a dramatic difference in the base case conditions by just adding $0.1 \mathrm{~mol} \%$ of p-xylene feed impurity into this column.

\subsection{With Addition of the Feed Tray Location as the Optimized Variables.}

If additionally feed tray location can be considered as another optimized variable in the overall optimization procedure, the base case condition is very different from that of Fig. 5. By considering feed tray location as another optimized variable does not defeat the original purpose of operating this column under both "no feed impurity" and "with feed impurity" cases. The optimization procedure will be more complicated by adding one more variable in the search algorithm. Basically the overall optimization procedure as in Section 3.1 can be followed to find the design condition with the lowest TAC at particular feed tray location. Then, this procedure is repeated to find the design condition with the lowest TAC at another feed tray location. By collecting all the results at many possible feed tray locations, the design condition with the minimized TAC can be obtained. The final optimized base case condition is summarized in Figure 6 with feed tray location changes from $9^{\text {th }}$ stage as in Fig. 5 to $24^{\text {th }}$ stage. The sidedraw location is also changed from $21^{\text {st }}$ stage as in Fig. 5 to $18^{\text {th }}$ stage. Notice also that the sidedraw flow rate is further reduced from $0.7 \mathrm{kmol} / \mathrm{h}$ to 0.2 $\mathrm{kmol} / \mathrm{h}$ because of much richer $\mathrm{p}$-xylene can be established inside the column at this new base case condition. The liquid composition profile for the base case of Fig. 6 is show in Figure 7.

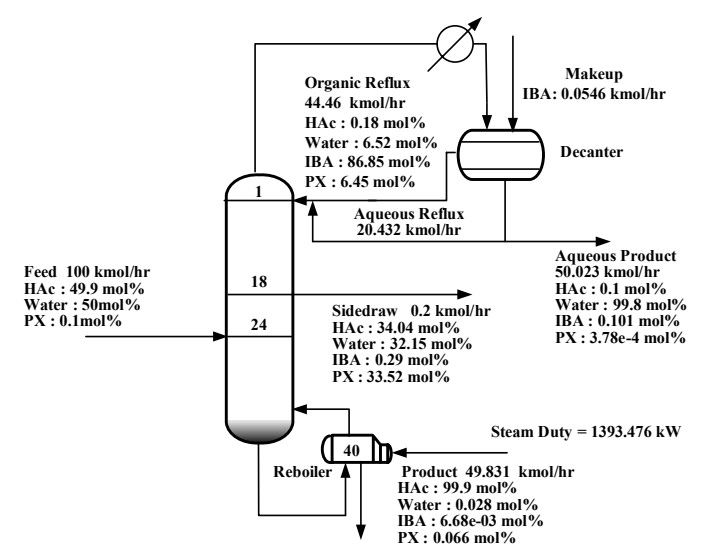

Fig. 6. Optimized base case condition with feed impurity (changing feed tray location)

By comparing Fig. 6 with earlier Fig. 5, it is found that the reboiler heat duty can be reduced from $1711 \mathrm{KW}$ to $1393 \mathrm{KW}$ (a $18.6 \%$ reduction). The TAC of the system is reduced from $\$ 559.5 \times 10^{3}$ to $\$ 438.3 \times 10^{3}$ (21.7\% reduction), and the operating cost is also reduced from $\$ 163.8 \times 10^{3}$ to $\$ 133.4 \times 10^{3}$ (18.6\% reduction). Hence, by just adding feed tray location as another variable in the optimization search, significant 
savings in TAC and operating energy can be realized. This base case design will be used in a following-up control study to investigate the proper overall control strategy to hold the bottom and top product purities despite feed water composition changes or the changes in p-xylene impurity in feed stream.

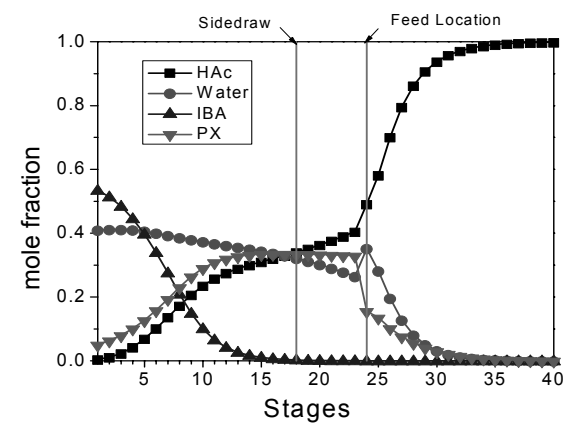

Fig. 7. Liquid composition profile for the base case of Fig. 6

\section{Conclusions}

In this paper, the process flowsheets with and without tiny impurity of $\mathrm{p}$-xylene are both considered in the optimal design of acetic acid dehydration column via heterogeneous azeotropic distillation. The simulation results show that dramatic difference in the base case conditions are found by just adding $0.1 \mathrm{~mol} \%$ of p-xylene feed impurity into this column. Over 50\% more TAC and operating energy are needed to operate this column with sidedraw under "with feed impurity" case. However, by considering feed tray location as an additional optimized variable in the optimization search, significant savings in TAC with a reduction of $21.7 \%$ can be realized. Note particularly with this change of the feed tray location from $9^{\text {th }}$ stage to $24^{\text {th }}$ stage, significant saving of the operating energy with a reduction of $18.6 \%$ can also be achieved.

\section{Acknowledgements}

This work is supported by the National Science Council of the R. O. C. under grant No: NSC 90-2214-E-011-013

\section{References}

[1] D. F. Othmer, Chem. Eng. Prog., 59, (1963) 67-78.

[2] H. N. Pham, and M. F. Doherty, Chem. Eng. Sci., 45, (1990) 1845-1854..

[3] S. Tanake, and J. Yamada, J. Chem. Eng. Jpn., 5, (1972) 20-26.

[4] D. F. Othmer, Chem. Metall. Eng., 40, (1941) 91-95.

[5] J. J. Siirola,In An Industrial Perspective on Process Synthesis; L. T. Bieglar, and M. F. Doherty, (eds.); AIChE Symposium Series 304; CACHE: Austin, TX, Vol. 91, (1995) pp $222-$ 233.

[6] S. K. Wasylkiewicz, L. C. Kobylka, and F. J. L. Castillo, Chem. Eng. J., 79, (2000) 219-227.

[7] I. L. Chien, K. L. Zeng, H. Y. Chao, J. H. Liu, Chem. Eng. Sci., 59, (2004) 4547-4567.

[8] I. L. Chien, H. P. Huang, T. K. Gau, and C. H. Wang, Ind. Eng. Chem. Res., 44, (2005) $3510-$ 3521.

[9] Aspen Technology, Inc. Aspen Plus User's Manual 11.1, Aspen Technology, Inc, Cambridge, MA, 2002. 